Pacific Journal of Mathematics

ON SEPARABLE POLYNOMIALS OVER A COMMUTATIVE 


\title{
ON SEPARABLE POLYNOMIALS OVER A COMMUTATIVE RING
}

F. R. DeMeyer

\begin{abstract}
Separable polynomials over an arbitrary commutative ring are studied. Given any separable polynomial $p(X)$ over the commutative ring $R$ one can find a "splitting ring" for $p(X)$ which is a finitely generated normal separable extension of $R$ generated by roots of $p(X)$. A polynomial closure $A$ of $R$ generated by roots of separable polynomials is constructed. Any separable polynomial over $\Lambda$ factors into linear factors in 1. A Galois theory for such extensions is discussed. Applications to separable extensions of von Neumann regular rings and the Brauer group are given.
\end{abstract}

In [6] G. J. Janusz developed the fundamental properties of separable polynomials over a commutative ring with no idempotents other than 0 and 1 . If $R$ is a commutative ring a monic polynomial $p(X)$ in $R[X]$ is separable in case $R[X] /(p(X))$ is a separable $R$-algebra. Here we develop a corresponding theory for separable polynomials over an arbitrary commutative ring. Since the fundamental difficulty to be overcome is the presence of idempotents, the tool (due to R. S. Pierce [11]) of representing a commutative ring as a global cross section of a sheaf of rings with no idempotents other than 0 and 1 is employed throughout. Basic properties of separable algebras and Galois theory discovered by A. Magid [8], [9], [10] are also employed.

Our first section is devoted to introducing necessary terminology and refining some of Magid's results for our own use. The principal result of the section is an extension of the fundamental theorem of Galois theory (Theorem 2.10 of [9]) to include the correspondence between normal extensions and normal subgroupoids of the Galois groupoid.

In the second section we analyze separable polynomials. Let $p(X)$ be a separable polynomial over the commutative ring $R$. We find a finitely generated normal separable extension $S$ of $R$ generated by roots of $p(X)$ and so that $p(X)$ factors into linear factors over $S$. Also, $S$ satisfies a "local projectivity" condition. Such an $R$-algebra $S$ is a splitting ring for $p(X)$. Associated to the extension $S$ of $R$ and thus to $p(X)$ is a compact totally disconnected topological groupoid $G(S / R)$. The nonuniqueness of $S$ and $G(S / R)$ are discussed. To each commutative ring $R$ we associate an extension $\Lambda$ called a polynomial closure of $R$ and a compact totally disconnected topological groupoid $G(\Lambda / R)$. The extension $\Lambda$ of $R$ is generated by roots of separable polynomials and every separable polynomial over $\Lambda$ factors into linear 
factors in $\Lambda$. We show that the Galois theory developed in [9] applies to $A$. The nonuniqueness of $\Lambda$ is also discussed.

In the third section we give applications of our results to separable extensions of von Neumann regular rings, to the Brauer group of rings whose prime ideal spectrum is totally disconnected, and to the Brauer group of the polynomial closure of such rings.

1. Preliminaries. Let $R$ be a commutative ring, $B(R)$ the Boolean algebra of idempotents of $R$, and $X(R)$ the maximal ideal spectrum of $B(R)$. Then $X(R)$ is a compact totally disconnect Hausdorff space in the hull-kernel topology. For each $x \in X(R)$ let $R x$ be the ideal in $R$ generated by $x$ and for each $R$-module $M$ let $M_{x}=M / R x M$. Then $R_{x}=R / R x$ and $M_{x}$ is an $R_{x}$-module. The rings $R_{x}$ are stalks in a sheaf over the base space $X(R)$ and $R$ is naturally represented as a global cross section of this sheaf. There are several standard lines of argument which have been developed to lift information true at all stalks $R_{x}$ to information about $R$. We will not usually go through the details of these arguments and refer the reader to [13], [3], [7], [12], among others for examples of this sort of reasoning.

In [9] A. Madgid calls a commutative $R$-algebra $S$ a quasi sepapable cover of $R$ in case for each $x \in X(R)$, every finite subset of $S_{x}$ is contained in a finitely generated projective separable subalgeba of $S_{x}$ ( $S_{x}$ is a locally strongly separable $R_{x}$-algebra). The algebra $S$ is a separable cover of $R$ if $S$ is a quasi separable cover of $R$ and $S$ is separable over $R$. Corollary 2.7 of [9] asserts that every quasi separable cover $S$ of $R$ is a union of subalgebras which are separable covers of $R$. $\quad R$ is called separably closed if whenever $S$ is any projective separable $R$-algebra then there is an $R$-algebra homomorphism from $S$ to $R$. A separably closed quasi separable cover $\Gamma$ of $R$ is called a separable closure of $R$. If $\Gamma$ is a separable closure of $R$ and $S$ is a projective separable $R$-algebra, then there is an $R$-algebra homomorphism from $S$ to $\Gamma$ induced from the one from $\Gamma \otimes_{R} S$ to $\Gamma$. This homomorphism need not be one-to-one and in general the separable closure need not be unique. The problem is in the existence of idempotents in $\Gamma \otimes_{R} S$ which are not in $\Gamma$.

If we call a ring $R$ extra separably closed in case whenever $S$ is a separable cover of $R$ which is finitely generated as an $R$-module then there is an $R$-algebra homomorphism from $S$ to $R$, then there is a unique minimal extra separable closure $S$ of $R$ [10]. The Boolean algebra $B(S)$ of idempotents of $S$ is the completion of $B(R)$. However, in many cases (for example if $X(R)$ is countable or $R$ is a uniform ring [9]) a separable closure of $R$ exists with no new idempotents. It is simpler to deal with the situation where no new idempotents are added so we will do this whenever possible (see Theorem 1.1). 
A quasi separable cover $S$ of $R$ is called normal in case for each $x \in X(R)$, all $R_{x}$-homomorphism from $S_{x}$ into the separable closure $\Gamma_{x}$ of $R_{x}$ have the same image. Since the composite of locally strongly separable $R_{x}$-subalgebras of an $R_{x}$ algebra $\Gamma$ with no idempotents other than 0 and 1 is locally strongly separable, this definition is equivalent to the one in [9]. Also, as observed in [8], $S$ is a normal quasi separable cover of $R$ if and only if whenever $x \in X(R)$ and $a, b \in X(S)$ with $a$ and $b$ lying over $x$ then $S_{a} \cong S_{b}$ as $R_{x}$-algebras and $S_{a}$ is a normal extension of $R_{x}$.

Our next step' is to restate and extend the fundamental theorem of Galois theory presented in [9]. Let $S$ be a normal quasi separable cover of $R$. Let $x \in X(R)$ and let $a, b \in X(S)$ lying over $x$. Let $h$ be an $R_{x}$-algebra isomorphism from $S_{b}$ to $S_{a}$. Let $\sigma$ be the set of idempotents in $S \otimes_{R} S$ contained in the kernel of the map $S \otimes_{R} S \rightarrow S_{a}$ by $s t \rightarrow s_{a} h\left(t_{b}\right)$. Then it is shown in [9] that the above correspondence gives a bijection between the points $\sigma$ of $X\left(S \otimes_{R} S\right)$ and the four tuples $(x, h, a, b)$ where $x \in X(R) ; a, b \in X(S)$ over $x$ and $h$ is an $R_{x}$-algebra isomorphism from $S_{b}$ to $S_{a}$.

The Galois groupoid $G$ of $S$ over $R$ is the set of four tuples $(x, g, a, b)$ with the topology corresponding to that in $X(S \otimes S)$. The partial multiplication is defined between pairs of four tuples of the following form,

$$
(x, g, a, b)(x, h, b, c)=(x, g h, a, c) .
$$

The identities of the groupoid are the four tuples $(x, 1, a, a)$ and a subgroupoid is a subset containing all identities and closed under multiplication and inversion.

Let $H$ be a subgroupoid of $G$. Let

$$
S^{H}=\left\{s \in S \mid g\left(s_{b}\right)=s_{a} \text { for all }(x, g, a, b) \in H\right\} .
$$

Let $T$ be an $R$-subalgebra of $S$. Let

$$
G(S / T)=\left\{(x, g, a, b) \in G \mid t_{a}=g\left(t_{b}\right) \text { for all } t \in T\right\} .
$$

Then Theorem 2.10 of [9] asserts the usual Galois correspondence between the set of all quasi separable covers of $R$ in $S$ and all closed subgroupoids of $G$. If $G$ and $H$ are groupoids and $h: G \rightarrow H$ is a homomorphism of $G$ onto $H$ then the inverse image of the identities of $H$ is a normal subgroupoid $K$ of $G$ and the natural multiplication on the quotient structure turns $G / K$ into a groupoid isomorphic to $H$. If $G$ and $H$ are compact Hausdorff topological groupoids and $h$ is continuous, then $K$ is closed in $G$ and the natural isomorphism from $G / K$ to $H$ is a homeomorphism. (See page. 16 of [1].)

Theorem 1.1. (Fundamental theorem of Galois theory.) Let $S$ 
be a normal quasi separable cover of $R$ with Galois groupoid $G$. Then there is a one-to-one correspondence between the quasi separable covers $T$ of $R$ in $S$ and the closed subgroupoids $K$ of $G$ by

$$
K \longrightarrow S^{K}, \quad T \longrightarrow G(S / T) \text {. }
$$

Moreover, if $T$ is a normal extension of $R$ then the corresponding subgroupoid $K$ is normal in $G$ and $G / K$ is the Galois groupoid of $T$ over $R$. If every idempotent of $S$ belongs to $R$ and $K$ is normal in $G$, then $S^{K}$ is a normal extension of $R$.

Proof. All except the moreover statement are proved in [9]. First we state a result which is a consequence of the Galois theory in Chapter III, $\S 3$ of [5].

Proposition 1.2. Let $R$ be a commutative ring whose only idempotents are 0 and 1 and let $\Gamma$ be the separable closure of $R$. Let $S$ be a normal locally strongly separable $R$-algebra whose only idempotents are 0 and 1 and let $T$ be a locally strongly separable $R$-subalgebra of $S$. Let $h$ be any $R$-homomorphism from $T$ to $\Gamma$, then $h$ can be extended to an $R$-monomorphism from $S$ to $\Gamma$. Any two extension of $h$ to $S$ have the same image in $\Gamma$. If $S$ is a subalgebra of $\Gamma$ then any extension of $h$ to $S$ is an automorphism of $S$.

Now we can prove the theorem. Assume $T$ is a normal extension of $R$ with Galois groupoid $K$. Define a groupoid homomorphism $h: G \rightarrow K$ by the rule

$$
h((x, g, a, b))=\left(x, g^{*}, a^{*}, b^{*}\right)
$$

where $x \in X(R) ; a, b \in X(S)$ lying over $x$, and $a^{*}, b^{*}$ are points in $X(T)$ so that $a$ lies over $a^{*}$ and $b$ lies over $b^{*}$. Also $g^{*}: T_{b^{*}} \rightarrow T_{a^{*}}$ by $g^{*}\left(t_{b^{*}}\right)=u_{a^{*}}$ in case $g\left(t_{b}\right)=u_{a}$ where $t, u \in T$. There is a natural $R_{x}$-homomorphism from $T_{a^{*}}$ into $S_{a}$ by $t_{a^{*}} \rightarrow t_{a}$. By Proposition 1.2 this correspondence is a monomorphism so if $t, v \in T$ then $t_{a}=v_{a}$ if and only if $t_{a^{*}}=v_{a^{*}}$. To see that $g^{*}$ is well defined assume $t, v \in T$ and $t_{b^{*}}=v_{b^{*}}$ in $T_{b^{*}}$. Then $t_{b}=v_{b}$ in $S_{b}$ so if $g\left(t_{b}\right)=u_{a}$ and $g\left(v_{b}\right)=w_{a}$ with $u, w \in T$ then $u_{a}=w_{a}$ since $g$ is well defined. Since $T$ is normal over $R$; $u, w$ can be chosen from $T$. Thus $u_{a^{*}}=w_{a^{*}}$ so $g^{*}$ is well defined. Now $T_{a^{*}}$ and $T_{b^{*}}$ are isomorphic locally strongly separable normal $R_{x}$-algebras with no idempotents other than 0 and 1 . By Proposition $1.2 g^{*}$ is an isomorphism. It is now routine to see that $h$ is a continuous homomorphism. Let $\left(x, g^{*}, a^{*}, b^{*}\right)$ be an element of $K$. Let $a$ and $b$ be elements of $X(R)$ lying over $a^{*}$ and $b^{*}$ respectively. Then $T_{a^{*}}$ is a locally strongly separable $R_{x^{-}}$-subalgebra of $S_{a}$ and $T_{b^{*}}$ is a 
locally strongly separable $R_{x}$-subalgebra of $S_{b}$. Also, $S_{a}$ and $S_{b}$ are isomorphic $R_{x}$-algebras which are normal over $R_{x}$. So by Proposition 1.2. $g^{*}$ extends to an isomorphism $g$ from $S_{b}$ to $S_{a}$. Thus, $\left(x, g^{*}, a^{*}, b^{*}\right)=$ $h((x, g, a, b))$ and $h$ is onto. The kernel of $h$ is the subgroupoid $H$ of $G$ which fixes $T$ and $G / H=K$.

Now assume every idempotent of $S$ belongs to $R$ and let $T$ be a locally quasi separable cover of $R$ in $S$. Let $K$ be the closed subgroupoid of $G$ with $S^{K}=T$ and assume $K$ is a normal subgroupoid of $G$. Since every idempotent of $S$ belongs to $R$, each element of $G$ is of the form $(x, g, x, x)$ where $g$ is an automorphism of $S_{x}$. Thus, $G$ is the disjoint union of the Galois groups of each $S_{x}$ over $R_{x}$ as $x$ ranges through $X(R)$ and multiplication is not defined between different terms in the union. One can show that $K$ must be the union of the normal subgroups which fix $T_{x}$ over $R_{x}$ in $S_{x}$. Thus, $T_{x}$ is a normal extension of $R_{x}$ for each $x \in X(R)$ so $T$ is a normal extension of $R$. This completes the proof.

If we do not assume that every idempotent in $S$ belongs to $R$ then for $a^{*}$ and $b^{*}$ in $X(T)$ lying over $x \in X(R)$ one may have $T_{a^{*}}$ and $T_{b^{*}}$ both normal over $R_{x}$ but not isomorphic. In this case $G(S / T)$ will be normal in $G(S / R)$ but $T$ will not be normal over $R$. If $\Gamma$ is a separable closure of $R$ and $a, b \in X(\Gamma)$ lying over $x \in X(R)$ then both $\Gamma_{a}$ and $\Gamma_{b}$ are separable closures of $R_{x}$. Any $R_{x}$-algebra homomorphism from $\Gamma_{a}$ and $\Gamma_{b}$ to a separable closure $\Omega$ of $R_{x}$ have image $\Omega$ (as is shown in the discussion on page 105 of [5]) so $\Gamma$ is normal over $R$.

2. Separable polynomials. Let $p(X)$ be a separable polynomial over $R$. A normal quasi separable cover $S$ of $R$ is called a splitting ring for $p(X)$ in case

(1) $S$ is generated over $R$ by roots of $p(X)$.

(2) $p(X)$ factors into linear factors in $S[X]$.

Proposition 2.1. Let $p(X) \in R[X]$ be a separable polynomial, then a splitting ring $S$ for $p(X)$ exists. Moreover, $S$ can be chosen to be finitely generated over $R$.

Proof. Let $T$ be a separable closure of $R$. Then $p(X)$ is separable over $\Gamma$ and there is an $\Gamma$-algebra homomorphism from $\Gamma[X] /(p(X))$ to $\Gamma$. The image of $X$ is a root $\alpha$ of $p(X)$ in $\Gamma$. Now $R_{x}\left(\alpha_{x}\right)$ is a finitely generated separable $R_{x}$-subalgebra of $\Gamma_{x}$ for each $x \in X(R)$ so by Lemma 1.2 of [8] $R(\alpha)$ is a finitely generated separable cover of $R$ in $\Gamma$. Let $T=R(\alpha)$. The transitivity properties of separability, projectivity, and finite generation insure that $\Gamma$ is a separable closure of $T$. Also, $p(X)=(X-\alpha) q(X)$ in $T[X]$ and $T[X] /(q(X))$ is a homomorphic image of $T[X] / p(X)$ by the natural map. Thus, $q(X)$ is a 
separable polynomial over $T$ and we can apply the first step in the proof to $q(X)$ over $T$. After a finite number of steps we come to an extension $S$ of $R$ which is a finitely generated separable cover of $R$, generated by roots of $p(X)$, and so that $p(X)$ factors into linear factors in $S$. Now let $x \in X(R)$ and $a, b \in X(S)$ lying over $x$. Then both $S_{a}$ and $S_{b}$ are splitting rings of the separable polynomial $p(X)$. By the normality and uniqueness of splitting rings in case of no nontrivial idempotents we have $S_{a}$ as a normal extension of $R_{x}$ and all homomorphisms from $S_{a}$ to $S_{b}$ to be a separable closure of $R_{x}$ have the same image. Thus, $S$ is normal over $R$.

With respect to uniqueness of splitting rings, the same situation holds as for the separable closure. Let $\Gamma$ be the separable closure of $R$ with the property that $B(\Gamma)$ is the completion of $B(R)$. Let $p(X)$ be a separable polynomial in $R[X]$ and let $S$ be the $R$-subalgebra of $\Gamma$ generated by all the roots of $p(X)$ in $\Gamma$. Then $S$ is a normal quasi separable cover of $R$ and any $R$-splitting ring $N$ of $p(X)$ with $B(N) \subseteq B(S)$ will be isomorphic to an $R$-subalgebra of $S$. If $B(R)$ is countable than $S$ can be chosen so that every idempotent in $S$ is in $R$ (see [9]). If $R$ is a uniform ring (see [4]) then in addition, $S$ can be chosen to be projective over $T$. Consider the following example. Let $X=\{1,1 / 2,1 / 3,1 / 4, \cdots 1 / n, \cdots\} \cup\{0\}$ with the topology inherited from the reals. Let $R$ be the ring of complex valued continuous $f$ on $X$ so that $f(0)$ is real with the discrete topology on the complex numbers. Let $S$ be the ring of continuous complex valued functions on $X$. Then $B(R)=B(S)$ and $S$ is a normal finitely generated separable cover of $R$. The Galois groupoid $G$ of $S$ over $R$ is the cyclic group of order 2 whose nonidentity element is complex conjugation at $f(0)$ for each $f \in S$. One observes that there are no nontrivial $R$ automorphisms of $S$, and there are no projective finitely generated separable covers of $R$ in $S$ containing a root of the separable polynomial $X^{2}+1$ in $R[X]$.

We next construct a normal quasi-separable cover $A$ of $R$ so that every separable polynomial over $R$ factors completely in $\Lambda$ and every finite subset of $\Lambda$ is contained in an extension $R\left(\alpha_{1}\right.$, $\left.\cdots, \alpha_{n}\right)$ of $R$ in $\Lambda$ with $\alpha_{i}$ the root of a separable polynomial in $R\left(\alpha_{1}, \cdots, \alpha_{i-1}\right)$. Such an extension $\Lambda$ is called a polynomial closure of $R$ (see [4]).

THEOREM 2.3. Let $R$ be a commutative ring, then a polynomial closure of $R$ exists and is a normal extension of $R$.

Proof. Let $\Gamma$ be a separable closure of $R$, consider the set $\mathscr{S}$ of all extensions of $R$ in $\Gamma$ of the form $R\left(\alpha_{1}, \cdots, \alpha_{n}\right)$ with $\alpha_{i}$ the root of a separable polynomial in $R\left(\alpha_{1}, \cdots, \alpha_{i-1}\right)$. The property of being 
a separable cover is transitive (2.3 of [9]) and as in the proof of Proposition 2.1 each $R\left(\alpha_{1}, \cdots, \alpha_{i}\right)$ is a separable cover of $R\left(\alpha_{1}, \cdots, \alpha_{i-1}\right)$ so $R\left(\alpha_{1}, \cdots, \alpha_{n}\right)$ is a separable cover of $R$. If $R\left(\beta_{1}, \cdots, \beta_{m}\right)$ is another element of $\mathscr{S}$, then the separable polynomial over $R$ having $\beta_{1}$ as a root is also separable over $R\left(\alpha_{1}, \cdots, \alpha_{n}\right)$ so $R\left(\alpha_{1}, \cdots, \alpha_{n}, \beta_{1}, \cdots \beta_{m}\right)$ is in $\mathscr{S}$. By a finite induction $R\left(\alpha_{1}, \cdots, \alpha_{n}, \beta_{1}, \cdots, \beta_{m}\right)$ is in $\mathscr{S}$ so $\mathscr{S}$ is a directed set under inclusion. Let $\Lambda$ be the union of all the elements in $\mathscr{S}$. Then $\Lambda$ is a quasi separable cover of $R$ in $\Gamma$. Let $p(X) \in A[X]$ be a separable polynomial and let $\left(t\left(Y^{i} Y^{j}\right)\right)$ be the $n \times n$ matrix whose $i+1, j+1$ entry is $Y^{i} Y^{j}$ where $Y=X+(p(X))$ in $\Lambda[X] / p(X), n=$ degree $(p(X))$, and $t$ is the trace of the free $\Lambda$-module $\Lambda[X] /(p(X))$. Exactly as in the proof of Theorem 4.4 page 111 of [5] one can show $p(X)$ is separable over $\Lambda$ if and only if $\operatorname{det}\left(t\left(Y^{i} Y^{j}\right)\right)$ is a unit in $\Lambda$. Find an element $S \in \mathscr{S}$ containing the coefficients of $p(X)$, the elements $t\left(Y^{i} Y^{j}\right)$ and $\operatorname{det}\left(t\left(Y^{i} Y^{j}\right)\right)^{ \pm 1}$. Then $p(X)$ is separable over $S$, and $\Gamma$ contains a root $\beta$ of $p(X)$ as proved in Proposition 2.1. Then $S(\beta) \in \mathscr{S}$ so $S(\beta) \subseteq \Lambda$ and $\Lambda$ contains a root $\beta$ of $p(X)$. Then in $\Lambda[X], p(X)=$ $(X-\beta) q(X)$ and $q(X)$ is separable over $\Lambda$. Continue the process until $p(X)$ factors completely in $\Lambda$. For normality first observe that $p(X)=X^{2}-X$ is separable over $R$ so every idempotent in $\Gamma$ is in 1. Let $x \in X(R)$ and let $a, b \in X(\Lambda)=X(\Gamma)$ be elements lying over $x$. Then $\Lambda_{a}$ and $\Lambda_{b}$ are polynomial closures of $R_{x}$ in the separable closures $\Gamma_{a}$ and $\Gamma_{b}$ of $R_{x}$. Therefore by Theorem 1.1 of [4], $\Lambda_{a}$ and $\Lambda_{b}$ are isomorphic normal extension of $R_{x}$ so $A$ is a normal extension of $R$.

We can take $B(A)$ to be the completion of $B(R)$ since every idempotent in $\Gamma$ is in $A$ and $B(\Gamma)$ can be the completion of $B(R)$. Also a compact totally disconnected groupoid $G(\Lambda / R)$ can be associated to the extension $A$ over $R$ which puts one in the context of the fundamental theorem of Galois theory, (Theorem 1.1).

3. Applications. A ring $R$ is von Neumann regular if and only if $R_{x}$ is a field for each $x \in X(R)$. On account of the primitive element theorem in the Galois theory of fields one has an extension of Theorem 2.7 of [4].

THEOREM 3.1. Let $R$ be a von Neumann regular ring and let $S$ be a finitely generated separable cover of $R$, then $S=R\left(\alpha_{1}, \cdots, \alpha_{n}\right)$ with $\alpha_{i}$ the root of a separable polynomial over $R\left(\alpha_{1}, \cdots, \alpha_{i-1}\right)$.

Proof. $\quad R$ is regular so for each $x \in X(R), R_{x}$ is a field. Therefore, $S_{x}$ is a finite direct sum of separable field extensions of $R_{x}$, say $S_{x}=F_{1} \oplus \cdots \oplus F_{n}$. By the primitive element theorem, $F_{1}=R_{x}\left(\alpha_{x}^{1}\right)$, $F_{2}=R\left(\alpha_{x}^{2}\right), \cdots, F_{n}=R\left(\alpha_{x}^{n}\right)$ with $\alpha_{x}^{i}$ satisfying separable polynomials 
$p_{x}^{i}(X)$ in $R_{x}[X]$ of the same degree (see [14]). Then $S_{x}=R_{x}\left(\alpha_{x}^{1}, \cdots, \alpha_{x}^{n}\right)$ and $\alpha_{i}^{x}$ satisfies the separable polynomial $p_{x}^{i}(X)$ in $R_{x}\left(\alpha_{x}^{1}, \cdots, \alpha_{x}^{i-1}\right)$. Lift $p_{x}^{i}(X)$ and $\alpha_{x}^{i}$ to monic polynomials $p^{i}(X) \in R[X]$ and $\alpha^{i} \in S$. Since $S_{x}=R_{x}\left(\alpha_{x}^{1}, \cdots, \alpha_{x}^{n}\right)$ and $S$ is finitely generated, (2.11) of [13] implies there is a neighborhood $U$ of $x$ so that for each $y \in U, S_{y}=$ $R_{y}\left(\alpha_{y}^{1}, \cdots, \alpha_{y}^{n}\right)$. Also, $p_{y}^{i}(X)$ is separable for each $y$ in a neighborhood of $x$. Therefore, there is an idempotent $e \in R$ so that $S e=$ $R e\left(\alpha_{n}^{1}, \cdots, \alpha_{n}^{n}\right)$ and $\alpha^{i} e$ satisfies the monic separable polynomial $p^{i}(X) e$ in $R e[X]$. Employing the compactness of $X(R)$ one can decompose $R$ by a finite number of such idempotents $e$ thereby obtaining the result.

COROLlaRY 3.2. If $R$ is a von Neumann regular ring then every separable closure of $R$ is a polynomial closure and conversely.

Let $\operatorname{Spec}(R)$ be the maximal ideal spectrum of $R$, than Spec $R$ is totally disconnected if and only if $R_{x}$ is semi-local (finite number of maximal ideals) for each $x \in X(R)$. For such rings we have the following result.

THEOREM 3.3. Let $R$ be a ring with Spec $(R)$ totally disconnected. If $A$ is a central separable $R$-algebra, then $A$ is split in the Brauer group of $R$ by a normal finitely generated separable cover $R\left(\alpha_{1}, \cdots, \alpha_{n}\right)$ of $R$ with $\alpha_{2}$ the root of a separable polynomial over $R\left(\alpha_{1}, \cdots, \alpha_{i-1}\right)$. If $\Lambda$ is a polynomial closure of $R$, we can assume $R\left(\alpha_{1}, \cdots, \alpha_{n}\right)$ is in $\Lambda$.

Proof. Modifying the proof of Theorem 1 in [2] by using Henselization instead of completion one has for each $x \in X(R)$ a strongly separable extension $S_{x}$ over $R_{x}$ of the form $R_{x}\left(\alpha_{x}\right)$ with $\alpha_{x}$ the root of a separable polynomial $p_{x}(X)$ over $R_{x}[X]$ which splits $A_{x}$. Now $S_{x}$ is contained in a normal separable extension $N_{x}$ of $R_{x}$ generated by the roots of $p_{x}(X)$ by Theorems 3.4.2 and 3.2.9 or [5]. Arguing as in Theorem 2.1 of [4] one can construct a finite set of orthogonal idempotents $e_{1}, \cdots, e_{n} \in R$ with $1=e_{1}+\cdots+e_{n}$ and $N e_{i}$ a normal extra separable extension of $R e_{2}$ in $\Lambda e_{i}$ generated by roots of the separable polynomial $p_{i}(X)$ and $N e_{i}$ splits $A e_{i}$. The extension $N=N e_{1} \oplus \cdots \oplus N e_{n}$ is the one we seek.

Lemma 3.4. Let $S$ be a locally extra separable $R$-algebra and $P$ a finitely generated projective $S$-module. Assume Spec $(R)$ is totally disconnected, then there is a finite set $e_{1}, \cdots, e_{n}$ of orthogonal idempotents in $S$ summing to 1 with $P e_{i}$ free over $S e_{i}$.

Proof. Let $x \in X(R)$ and let $a \in X(S)$ lying over $x$. Then $S_{a}$ is a locally strongly separable $R_{x}$-algebra with no idempotents other than 0 and 1 and $R_{x}$ is a semi-local ring so the proof of Proposition 3 of 
[2] shows $P_{a}$ is a free $S_{a}$-module. Let $\left[P_{a}: S_{a}\right]$ be the rank of $P_{a}$ over $S_{a}$. The function $a \rightarrow\left[P_{a}: S_{a}\right]$ is continuous and bounded from $X(S)$ to the nonnegative integers so there exists orthogonal idempotents $e_{1}, \cdots, e_{n}$ in $S$ summing to 1 with the rank of $P e_{i}$ over $S e_{i}$ defined. Let $P=P e_{\imath}$ and $S=S e_{i}$ with $[P: S]=n$. Let $a \in X(S)$ and let $y_{a}^{1}$, $\cdots, y_{a}^{n}$ be a free basis for $P_{a}$ over $S_{a}$. Lift the $y_{a}^{i}$ to elements $y^{i}$ in $P$, and let $E$ be the submodule of $P$ generated by $y^{i}$. Then $E_{a}=P_{a}$ so $E_{b}=P_{b}$ for all $b$ in a neighborhood of $a$ (2.11 of [13]). This neighborhood determines an idempotent $e$ of $S$ and $e y^{1}$ generate $P e$ over $R e$. The natural map from $P e$ onto the free $S e$-module $S e^{(n)}$ by assigning the $e y^{i}$ to basis elements of $S e^{(n)}$ has a kernel $K$ which is a direct summand of $P$. Now $K_{a}=0$ so in a neighborhood $V$ of $a, K_{y}=0$ for each $y \in V$. This gives an idempotent $e_{1} \in S e$ with $P e_{1}$ free over $S e_{1}$. Using the compactness of $X(S)$ gives the result.

COROLlary 3.5. Let $\Lambda$ be a polynomial closure or a separable closure of $R$. If Spec $(R)$ is totally disconnected, then the Brauer group of $\Lambda$ is trivial.

Proof. Let $A$ be a central separable $\Lambda$-algebra. We can write $A=A e_{1} \oplus \cdots \oplus A e_{n}$ over $\Lambda e_{1} \oplus \cdots \oplus \Lambda e_{n}$ with $A e_{i}$ a free $\Lambda e_{i}$-module. Let $a_{1}^{i}, \cdots, a_{n}^{i}$ be a free $\Lambda e_{i}$ basis for $A e_{i}$. Let $c_{l j}^{k i}$ be the multiplication constants for the algebra $A$ with respect to the basis $a_{1}^{i}, \cdots, a_{n}^{i}$, that is $a_{l}^{i} a_{m}^{2}=\Sigma_{k} c_{l m}^{k i} a_{k}^{i}$ with $c_{l m}^{k i} \in \Lambda$. Let $S=R\left(\alpha_{1}, \cdots, \alpha_{n}\right)$ be a finitely generated separable cover of $R$ in $\Lambda$ containing $\left\{c_{l m}^{k i}, e_{j}\right\}$. Define the central separable $S$-algebra $A_{S}$ by letting $A_{S} e_{j}$ be the free $S e_{j}$-module $S e_{j} a_{1}^{i}+$ $\cdots+S e_{j} a_{n}^{i}$ with multiplication constants $\left\{c_{l m}^{k i}\right\}$.

Now Spec $(R)$ is totally disconnected and since $S$ is a finitely generated separable cover of $R$, Spec $(S)$ is totally disconnected. By Theorem 3.3 there is a finitely generated separable over $S\left(\beta_{1}, \cdots, \beta_{m}\right)$ of $S$ in $A$ which splits $A$. Let $T=S\left(\beta_{1}, \cdots, \beta_{m}\right)$ then $A \cong \Lambda \bigotimes_{T}\left(T \otimes_{S} A_{S}\right)$ so $A$ is in the zero class of the Brauer group of $\Lambda$. The argument in case $A$ is a separable closure of $R$ is completely analogous.

As an alternate proof of the last result, we can use Theorem 1.1 of [4]. One can show as in Proposition 3.3 of [9] that a quasi separable cover $A$ of $R$ is a polynomial closure of $R$ if and only if for each $x \in X(R)$ and each $a \in X(\Lambda)$ lying over $x$ that $\Lambda_{a}$ is a polynomial closure of $R_{x}$. Corollary 1.11 of [7] asserts that the Brauer group of $\Lambda$ is trivial if and only if the Brauer group of $\Lambda_{a}$ it trivial for each $a \in X(\Lambda)$. Thus, an alternate proof of Corollary 3.5 would be provided by showing the Brauer group of the polynomial closure of a semi-local ring whose only idempotents are 0 and 1 is trivial. This is proved in Theorem 1.6 of [12]. 


\section{REFERENCES}

1. A. H. Clifford and G. B. Preston, The Algebraic Theory of Semi groups, Amer. Math. Soc. Math. Surveys, No. 7 (1961).

2. F. R. DeMeyer, The Brauer groups of some separably closed rings, Osaka J. Math., 3 (1966), 201-204.

3. - On automorphisms of separable algebras II, Pacific J. Math., 32 (1970), $621-631$.

4. - Separable polynomials over a commutative ring, Rocky Mountain J. Math., 2 (1972), 299-310.

5. F. R. DeMeyer, E. C. Ingraham, Separable Algebras over a Commutative Rings Lecture Notes in Mathematics, No. 181, Springer-Verlag, New York, 1971.

6. G. J. Janusz, Separable algebras over commutative rings, Trans. Amer. Math. Soc., 122 (1966), 461-479.

7. A Magid, Pierce's representation and separable algebras, Illinois J. Math., 15 (1971), $114-121$.

8. — Galois groupoids, J. Algebra, 18 (1971), 89-102.

9. - The separable closure of some commutative rings, Trans. Amer. Math. Soc., 170 (1972), 109-124.

10. - The fundamental groupoid of a commutative ring, to appear.

11. R. S. Pierce, Modules over commutative regular rings, Mem. Amer. Math. Soc., No. 70 (1967).

12. G. Szeto, The Azumaya algebra of some polynomial closed rings, Indiana University Math. J., to appear.

13. O. F. Villamayor and D. Zelinsky, Galois theory with infinitely many idempotents, Nagoya Math. J., 35 (1969), 83-98.

14. T. Nagahara, On a theorem of F. DeMeyer, Proc. Japan Acad., 49 (1973), 681-684.

Received November 8, 1972.

COLORADO STATE UNIVERSITY 


\section{PACIFIC JOURNAL OF MATHEMATICS}

\section{EDITORS}

RICHARD ARENS (Managing Editor)

University of California

Los Angeles, California 90024

R. A. BeAumont

University of Washington

Seattle, Washington 98105
J. DugundJI*

Department of Mathematics

University of Southern California

Los Angeles, California 90007

D. Gilbarg and J. Milgram

Stanford University

Stanford, California 94305

\section{ASSOCIATE EDITORS}

E. F. BECKENBACH

B. H. NEUMANN

F. WOLF

K. YoSHIDA

\section{SUPPORTING INSTITUTIONS}

UNIVERSITY OF BRITISH COLUMBIA
CALIFORNIA INSTITUTE OF TECHNOLOGY
UNIVERSITY OF CALIFORNIA
MONTANA STATE UNIVERSITY
UNIVERSITY OF NEVADA
NEW MEXICO STATE UNIVERSITY
OREGON STATE UNIVERSITY
UNIVERSITY OF OREGON
OSAKA UNIVERSITY

UNIVERSITY OF BRITISH COLUMBIA CALIFORNIA INSTITUTE OF TECHNOLOGY UNIVERSITY OF CALIFORNIA MONTANA STATE UNIVERSITY NEW MEXICO STATE UNIVERSITY UNIVERSITY OF OREGON OSAKA UNIVERSITY
UNIVERSITY OF SOUTHERN CALIFORNIA STANFORD UNIVERSITY UNIVERSITY OF TOKYO UNIVERSITY OF UTAH WASHINGTON STATE UNIVERSITY UNIVERSITY OF WASHINGTON AMERICAN MATHEMATICAL SOCIETY NAVAL WEAPONS CENTER

* C. R. DePrima California Institute of Technology, Pasadena, CA 91109, will replace J. Dugundji until August 1974. 


\section{Pacific Journal of Mathematics}

\section{Vol. 51, No. $1 \quad$ November, 1974}

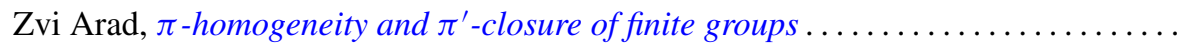

Ivan Baggs, A connected Hausdorff space which is not contained in a maximal

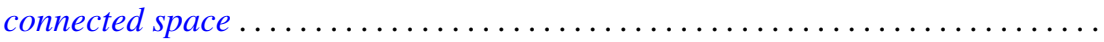

Eric Bedford, The Dirichlet problem for some overdetermined systems on the unit ball in $C^{n}$

R. H. Bing, Woodrow Wilson Bledsoe and R. Daniel Mauldin, Sets generated by

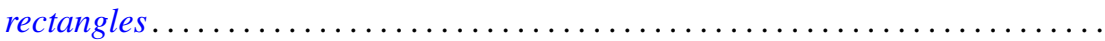

Carlo Cecchini and Alessandro Figà-Talamanca, Projections of uniqueness for

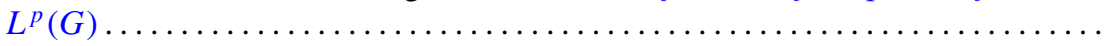

Gokulananda Das and Ram N. Mohapatra, The non absolute Nörlund summability of Fourier series .

Frank Rimi DeMeyer, On separable polynomials over a commutative ring ........ Richard Detmer, Sets which are tame in arcs in $E^{3} \ldots \ldots \ldots \ldots \ldots \ldots \ldots \ldots$

William Erb Dietrich, Ideals in convolution algebras on Abelian groups ..........

Bryce L. Elkins, A Galois theory for linear topological rings .................

William Alan Feldman, A characterization of the topology of compact convergence on $C(X)$.

Hillel Halkin Gershenson, A problem in compact Lie groups and framed cobordism

Samuel R. Gordon, Associators in simple algebras.

Marvin J. Greenberg, Strictly local solutions of Diophantine equations

Jon Craig Helton, Product integrals and inverses in normed rings . . . . . . . . . . . .

Domingo Antonio Herrero, Inner functions under uniform topology . . .

Jerry Alan Johnson, Lipschitz spaces .

Marvin Stanford Keener, Oscillatory solutions and multi-point boundary value

functions for certain nth-order linear ordinary differential equations.

John Cronan Kieffer, A simple proof of the Moy-Perez generalization of the

Shannon-McMillan theorem .......................

Joong Ho Kim, Power invariant rings

Gangaram S. Ladde and V. Lakshmikantham, On flow-invariant sets .

Roger T. Lewis, Oscillation and nonoscillation criteria for some self-adjoint even

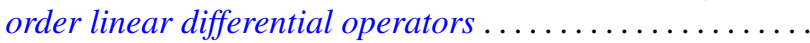

Jürg Thomas Marti, On the existence of support points of solid convex sets ..

John Rowlay Martin, Determining knot types from diagrams of knots . .

James Jerome Metzger, Local ideals in a topological algebra of entire functions

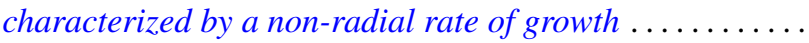

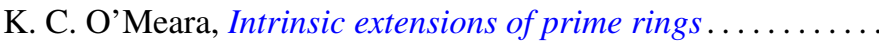

Stanley Poreda, A note on the continuity of best polynomial approximations ..

Robert John Sacker, Asymptotic approach to periodic orbits and local prolongations

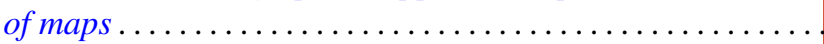

Eric Peter Smith, The Garabedian function of an arbitrary compact set . .

Arne Stray, Pointwise bounded approximation by functions satisfying a side condition

John St. Clair Werth, Jr., Maximal pure subgroups of torsion complete abelian

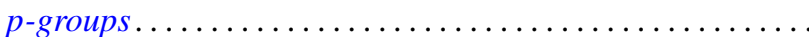

